
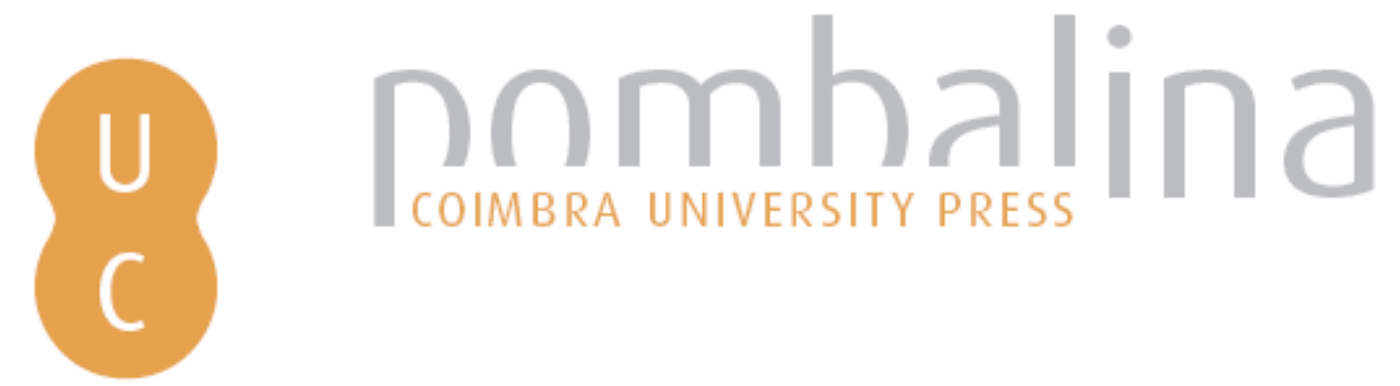

\title{
Las rutas historiográficas de la red de intercâmbios para la historia y la epistemologia de las ciências químicas y biológicas
}

Autor(es): $\quad$ Pastrana, Patricia Aceves

Publicado por: Imprensa da Universidade de Coimbra

URL

persistente: URI:http://hdl.handle.net/10316.2/32364

DOI: $\quad$ DOI:http://dx.doi.org/10.14195/978-989-26-0469-5_1

Accessed : $\quad$ 26-Apr-2023 06:14:06

A navegação consulta e descarregamento dos títulos inseridos nas Bibliotecas Digitais UC Digitalis, UC Pombalina e UC Impactum, pressupõem a aceitação plena e sem reservas dos Termos e Condições de Uso destas Bibliotecas Digitais, disponíveis em https://digitalis.uc.pt/pt-pt/termos.

Conforme exposto nos referidos Termos e Condições de Uso, o descarregamento de títulos de acesso restrito requer uma licença válida de autorização devendo o utilizador aceder ao(s) documento(s) a partir de um endereço de IP da instituição detentora da supramencionada licença.

Ao utilizador é apenas permitido o descarregamento para uso pessoal, pelo que o emprego do(s) título(s) descarregado(s) para outro fim, designadamente comercial, carece de autorização do respetivo autor ou editor da obra.

Na medida em que todas as obras da UC Digitalis se encontram protegidas pelo Código do Direito de Autor e Direitos Conexos e demais legislação aplicável, toda a cópia, parcial ou total, deste documento, nos casos em que é legalmente admitida, deverá conter ou fazer-se acompanhar por este aviso.

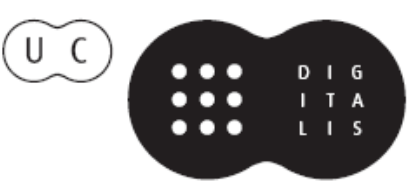


Ana Leunar Pereira João Rui Pita

[ Coordenaçä̃ ]
Rotas da Natureza

Cientistas

Viagens

Expedifgũes

Instituip̧ües

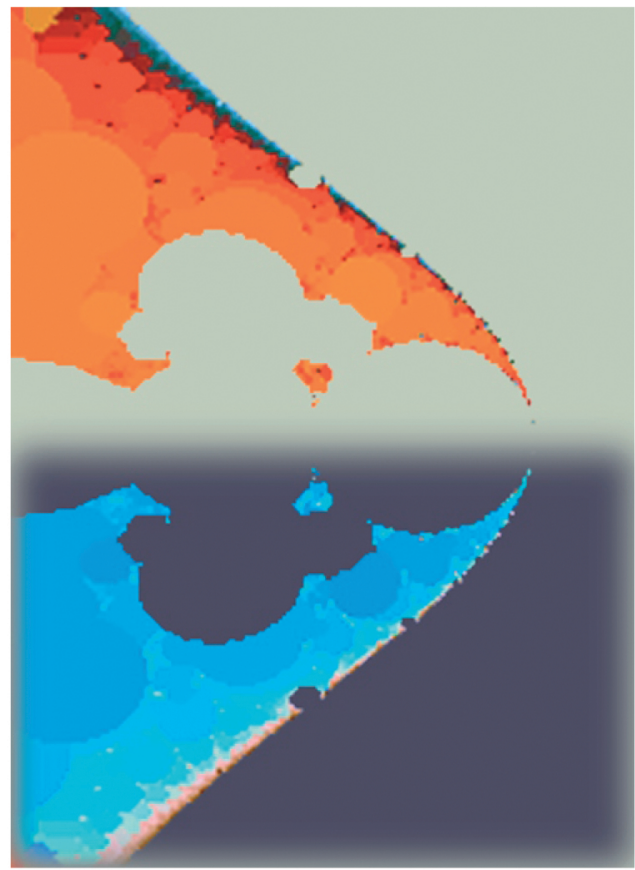




\section{Coordenaçáo Científica da Colecção Ciências e Culturas}

João Rui Pita e Ana Leonor Pereira

Os originais enviados são sujeitos a apreciação científica por referees

\section{Coordenação Editorial}

Maria João Padez Ferreira de Castro

\section{Edição}

Imprensa da Universidade de Coimbra

Email:impresauc@ci.uc.pt

URL: http://www.imp.uc.pt • Normas de publicação de colecçôes

\section{Design}

António Barros

Pré-Impressáo

António Resende

Imprensa da Universidade de Coimbra

\section{Capa}

António Barros, com imagem de E. M. de Melo e Castro, 2003 [Fractal original gerado no Fractint com tratamento no Photoshop 7.0]; Cortesia: António Barros

Impressão e Acabamento

SerSilito • Maia

\section{ISBN}

978-989-8074-12-6

\section{Depósito Legal}

Obra publicada com a colaboraçáo de:
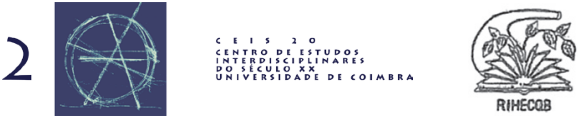

Obra publicada com o apoio de:

FCT Fundação para a Ciência e a Tecnologia

MINISTÉRIO DA CIÊNCIA, TECNOLOGIA E ENSINO SUPERTOR Portug

Programa Operacional Ciência, Tecnologia, Inovação do Quadro Comunitário de Apoio III
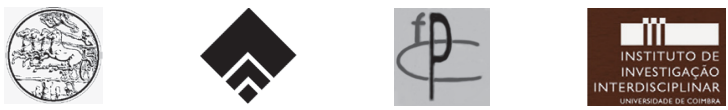

\section{Baxter}

(c) 2006, Imprensa da Universidade de Coimbra 
João Rui Pita

Ana Leonar Pereira

(Courdenação)

Rotas da Natureza

Cientistas

Viagens

Expediçũes

Instituiç̃̃es

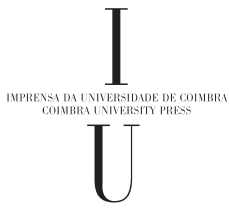

- colmbra 2006 
(Página deixada propositadamente em branco) 


\section{Patricia Aceves Pastrana}

Universidad Autónoma Metropolitana - Unidad Xochimilco, México

\section{LAS RUTAS HISTORIOGRÁFICAS DE LA RED DE INTERCÂMBIOS PARA LA HISTORIA Y LA EPISTEMOLOGIA DE LAS CIÊNCIAS QUÍMICAS Y BIOLÓGICAS}

\section{Introducción}

Queridas y queridos colegas, a nombre de los miembros de la Red de Intercambios para la Historia y la Epistemología de las Ciencias Químicas y Biológicas (RIHECQB), para mí es un honor pronunciar la conferencia de apertura en esta solemne y feliz sesión inaugural de los trabajos de la XII Reunión de la RIHECQB. Antes de entrar en materia, deseo agradecer encarecidamente a los organizadores de este evento por todos los desvelos pasados y por pasar, para recibirnos en Portugal; en especial a la Dra. Ana Luisa Janeira y al Comité Organizador encabezado por los doctores Isabel Cruz, Jose Augusto Mourao, Ana Leonor Pereira y Joao Rui Pita. También deseo dar las gracias a las instituciones que nos han acogido: el Centro Interdisciplinario de Ciencia Tecnología y Sociedad de la Universidad de Lisboa, el Centro Interdisciplinario del Siglo XX de la Universidad de Coimbra y el grupo de Historia y Sociología de la Ciencia de esta última. Asimismo reciban nuestro reconocimiento, todos aquellos que han respondido a la convocatoria de los organizadores.

En los últimos nueve años, la RIHECQB ha logrado publicar los resultados de sus investigaciones en diez volúmenes: siete en la Colección Estudios de Historia Social de las Ciencias Quimicas y Biológicas, dos en los Anais da Sociedade Brasileira de História da Ciencia, y uno en la Collection de Travaux de l'Académie Internationale d'Histoire des Sciences. Esta pequeña biblioteca representa varios millares de páginas que recogen cientos de contribuciones elaboradas por investigadores provenientes principalmente de once países: México, Brasil, Argentina, Venezuela, España, Portugal, Francia, Inglaterra, Bélgica, Australia y Estados Unidos.

A lo largo de su primera década de existencia, la RIHECQB ha podido consolidar sus líneas de investigación y sus espacios académicos. En la actualidad los distintos grupos de investigación que la conforman, han sabido crear escuela en sus respectivos países, además de establecer y extender fructíferos intercambios con la comunidad internacional. A través de años de intensa actividad, los integrantes de la Red hemos aprendido de todos y también hemos tenido tiempo para iniciar y refrendar una y otra vez, profundos lazos 
de amistad, respeto y confianza; sobre la base de las ininterrumpidas reuniones que año con año hemos efectuado en diversos lugares.

Hoy, que conmemoramos la primera Reunión de nuestra Red realizada en esta bella ciudad de Lisboa hace casi diez años, vale la pena recordar parte de la memoria que en conjunto hemos construido y que alegres festejaremos durante los días de este feliz reencuentro.

\section{Antecedentes}

La Red de Intercambios para la Historia y la Epistemología de las Ciencias Químicas y Biológicas surgió en 1992 por la iniciativa de un grupo de académicos afiliados a la Sociedad Latinoamericana de Historia de la Ciencia y la Tecnología, que fuimos invitados por la Dra. Ana María Alfonso Goldfarb al «Congreso América 92» realizado en Sao Paulo Brasil y Rio de Janeiro en agosto de 1992. Durante la semana que duró este magno evento varios historiadores de las ciencias químicas y farmacéuticas reflexionamos sobre la urgente necesidad de construir un lugar común de encuentro y los mecanismos para establecer la comunicación y el intercambio de información entre los investigadores provenientes de disciplinas diversas y de distintas regiones geográficas; con el fin de integrar una visión de conjunto del desarrollo de estos campos.

Así las cosas, el último día del Congreso ante un suculento plato de Bacalao pudimos concretar el proyecto de lo que bautizamos como la Red de Intercambios para la Historia y la Epistemología de las Ciencias Químicas y Biológicas, siendo sus progenitoras y madrinas: las doctoras Ana María Alfonso Goldfarb y Marcia Mendes Ferraz de Brasil, la Dra. Celina Lértora de Argentina, la Dra. Ana Luisa Janeira de Portugal y por México, una servidora. En aquel momento también decidimos integrar a la directiva de la Red al Dr. Javier Puerto Sarmiento de España, al Dr. Pedro Pruna de Cuba y a la Dra. Hebe Vesuri de Venezuela. Si bien, en la práctica nunca fue posible incorporar a estos dos últimos profesores, en el camino se nos unió la Dra. Luzia Aurelia Castañeda de Brasil y el Dr. Patrice Bret de Francia.

La agenda de trabajo para el futuro cercano incluía dar a conocer esta Red en nuestras instituciones y países, además de organizar dos eventos académicos, para reencontrarnos en el verano de 1993. Así lo hicimos y al siguiente año pudimos reunirnos, en el mes de agosto, por iniciativa de la Dra. Alfonso Goldfarb, en el Simposio "Historia de los estudios médicos y químicos de las aguas minerales (siglos XVI-XIX)» dentro del Congreso Internacional de Historia de la Ciencia realizado en Zaragoza España. Pocos días después, nos veríamos nuevamente en la primera Reunión la RIHECQB realizada en Lisboa el 2 de septiembre de 1993, misma de la que hoy conmemoramos su $\mathrm{X}$ aniversario.

A partir de ese momento comenzaría el desarrollo de un sueño y de un proyecto académico, cuyos resultados superarían con creces nuestras expectativas iniciales; aun a pesar del paso de los años transcurridos, las distancias por recorrer y nuestras personalidades diversas. En los minutos que me restan presentaré un resumen apretado de los objetivos, desarrollo, mecanismos de acción, resultados y balance historiográfico de las publicaciones de la Red. 


\section{La estructura de la RIHECQB}

Desde el inicio de nuestra Red, los investigadores que la integramos hemos organizado su actividad sobre la base de los objetivos siguientes:

- Vincular a los grupos de investigación regionales e internacionales interesados en la historia y la epistemología de las ciencias químicas y biológicas.

- Promover la comunicación y el intercambio de información entre sus miembros.

- Proporcionar a los investigadores un foro de análisis y discusión de los estudios generados en este campo.

- Organizar reuniones académicas para la presentación de los avances de investigación.

- Promover la realización de publicaciones temáticas.

Al mismo tiempo y con el propósito de agrupar a sus miembros y facilitar los intercambios entre ellos se establecieron los nudos temáticos que ha continuación se mencionan:

- La materia y sus transformaciones: aspectos filosóficos, sociológicos e históricos.

- Intercambios científico-técnicos en la metalurgia y en la mineralogía.

- Industria y ciencias químicas y biológicas.

- Sanidad y salud.

- Naturaleza y sociedad.

Comunicación y semiótica de las ciencias químicas y biológicas (representaciones, espacios y discursos).

- Sociedad, ciencia y valores.

Debido a que las temáticas precedentes son cultivadas por un número considerable de académicos, paulatinamente y de manera natural se ha ido incrementando el número de investigadores participantes en la Red.

En cuanto al Consejo Directivo de la RIHECQB, ha estado integrado con los académicos que se mencionan enseguida:

Coordinadora General: Dra. Patricia Aceves, México.

Coordinadores por país:

Dra. Celina Lértora, Argentina.

Dra. Ana María Alfonso Goldfarb, Brasil.

Dr. Francisco Javier Puerto Sarmiento, España.

Dr. Patrice Bret,Francia.

Dra. Ana Luisa Janeira, Portugal.

\section{Los estudios comparativos: la realización de un proyecto}

Con el fin de dar cumplimiento a los objetivos de la RIHECQB, año tras año el Consejo Directivo acordó la temática eje para la próxima reunión; temática que también articuló los trabajos a integrar en la publicación siguiente. Así, con doce meses o más de antici- 
pación los distintos grupos de investigación participantes unen sus esfuerzos en torno a un proyecto común y asumen el compromiso de discutir los resultados obtenidos en el próximo encuentro. Esta dinámica de trabajo renovada una y otra vez, nos ha permitido aprovechar la capacidad de los miembros de la RIHECQB, extender nuestras redes, madurar en paralelo y elaborar publicaciones de gran calidad.

Hasta el momento los intereses de investigación de la RIHECQB, publicados en diez volúmenes, han girado principalmente alrededor de dos grandes áreas del conocimiento:

- La historia de las ciencias, las ideas y las técnicas químicas y biológicas.

- La historia de los saberes profesionales y populares sobre las enfermedades y las terapias para mantener el cuerpo y el alma sanos ${ }^{1}$.

Una característica singular de nuestra pequeña biblioteca es que trata de recuperar la gran diversidad cultural alrededor de temas comunes, en un esfuerzo por explicar los intercambios científicos y culturales entre las metrópolis y sus colonias, así como sus influencias recíprocas tanto positivas como negativas. Este ha sido un intento exitoso de integrar la historia del espacio iberoamericano, que en muchos de los casos ha desbordado sus fronteras y abarca otras regiones.

Las investigaciones contenidas en los dos grandes apartados mencionados, presentan múltiples caras y ramificaciones hacia la farmacia, la medicina, la química, la biología, la metalurgia y la industria; y nos permiten recorrer un territorio, donde la multiplicidad rebasa toda definición a priori, y nos conduce a historias en proceso de hacerse, que se parecen menos a la marcha triunfal y positiva de la historiografía tradicional de las ciencias. Historias plurales que nos hablan de ciencias tremendamente viejas y modernas a la vez, con sus éxitos y equivocaciones. Herederas de las técnicas más antiguas que han definido a la humanidad y que continúan haciendo aportes de primera línea ${ }^{2}$.

Este espacio historiográfico dilata aún más sus alcances si tomamos en cuenta su extensión en el tiempo. Si bien una gran parte de las contribuciones aluden a los siglos XVIII y XIX, el resto se refiere a otros periodos históricos más remotos: la antigüedad grecolatina, la Edad Media, las culturas prehispánicas, el Renacimiento y las revoluciones científicas del siglo XVII.

\section{Las tematicas analizadas}

La mayoría de las temáticas expuestas por los autores pueden caer en las divisiones siguientes:

- Fuentes para la historia de las ciencias químicas y biológicas en Europa y América.

- Materia médica y farmacia de la antigüedad al siglo XIX.

- Tradición e innovación en los saberes teóricos prácticos y técnicos.

\footnotetext{
${ }^{1}$ Retomo estas dos grandes áreas del conocimiento y algunas ideas del Prólogo escrito por mi colega el Dr. Adolfo Olea Franco para el volumen 5 de los Estudios e Historia Social de la ciencias Químicas y Biológicas.

${ }^{2}$ Bensaude-Vincent, Bernadette, Histoire de la chimie, Paris, Éditions La Découverte, 1993.
} 
- Introducción, adopción y aplicación de los postulados de la química moderna en territorio iberoamericano, en particular en el terreno de la medicina, la farmacia y la metalurgia.

- Procesos de profesionalización e institucionalización de la química y la farmacia en los siglos XVII al XIX,

La geografía del saber dibujada en las distintas publicaciones de la RIHECQB, refleja la gran riqueza de las alternativas historiográficas presentadas por los autores y proporciona varios planos de interpretación donde se superpone lo mundial, lo nacional y lo regional.

Por un lado, la historia de las prácticas instrumentales y los oficios nos permite rastrear de qué manera las ciencias van conformando sus objetos de estudio a partir de un conjunto de operaciones y técnicas instrumentales. En un movimiento complementario, podemos analizar cómo los discursos epistemológicos forjados por los científicos van legitimando sus prácticas experimentales. Desde otro ángulo, el estudio de las ideas y los conceptos que integran una teoría no tiene por qué ser contrario a preguntarse por las condiciones materiales y culturales que las posibilitaron, a entender el contexto social, político y económico donde se originaron.

En los volúmenes editados, a través de la obra de científicos prominentes se puede acceder a un colectivo, definido no solamente por sus anclajes institucionales, sino también por sus prácticas comunes de laboratorio y de lenguaje. Junto a estos grandes nombres, también aparecen personajes de la historia local que ayudaron a forjar las tradiciones e instituciones científicas y que nos permiten ahondar en las particularidades de cada caso. De este modo, al revisar las distintas tradiciones de investigación, uno cae en la cuenta de que en cada época no existe un consenso de opiniones y una homogeneidad de prácticas. Sin lugar a dudas, la situación se rebela más compleja para la historia de las ciencias médicas, farmacéuticas, químicas y biológicas, donde en diferentes momentos coexisten en tensión teorías y prácticas antiguas y modernas. Para seguir de cerca los conflictos y negociaciones implícitos en estas tensiones y escribir una historia más humana y real, es necesario dejar atrás la tentación de simplificarlo todo a la victoria de un paradigma sobre otro, ya que ninguna teoría científica se impone por ella misma, como una luz que ahuyenta la tinieblas.

Por último, la historia de las instituciones nos sitúa en la nivel donde se define la cientificidad de las ciencias y se reproduce su cultura. La creación de nuevas instituciones, periódicos científicos, convenciones de nomenclatura, reglamentos y premios, son elementos que contribuyen al establecimiento de una ciencia académica, y a su legitimación social y política entre las prácticas sociales.

En resumen, el conjunto de los trabajos que integran los volúmenes de la RIHECQB nos permiten entender las manifestaciones científicas y culturales desarrolladas en los nuevos países surgidos a sangre y fuego a partir de la llegada de los europeos a tierras americanas, y delinean una gama de problemáticas que bajo distintos enfoques presentan diferentes matices de la reflexión histórica, epistemológica y sociológica. El amplio espectro del espacio temático permite acceder a diferentes aspectos del quehacer científico. Por un lado están las teorías científicas, la bibliografía y los desafíos científico-técnicos del momento; por el otro, podemos encontrar las técnicas y los métodos utilizados en el laboratorio y los aspectos técnicos de la incipiente industria química. Asimismo, no 
podían quedar fuera las expediciones y comunidades científicas, los centros de enseñanza e investigación y el papel del Estado en estos ámbitos. Por último, también se evidencia la vinculación existente entre los campos de la química, la medicina, la botánica, la farmacia, la mineralogía y la metalurgia.

\section{Las metas logradas}

En estos diez años los miembros de la RIHECQB hemos acumulado un paquete importante de logros y aportaciones. Á continuación se citan los más significativos.

1. Publicación de estudios temáticos comparativos de historia de las ciencias químicas, farmacéuticas y biológicas.

2. Formación de recursos humanos en estas áreas mediante la dirección de tesis de grado y posgrado en historia de la ciencia.

3. Apertura de nuevos espacios académicos, tales como proyectos y grupos de investigación, así como programas de estudio en Historia de la Ciencia de grado y posgrado.

4. Socialización de los resultados de investigación en diversos foros académicos y publicaciones especializadas.

5. Consolidación en el ámbito internacional del grupo de investigadores que conforman la RIHECQB y de sus diversas publicaciones. En esta línea de acción se realizaron once reuniones internacionales, cinco Simposia internacionales y dos cursos de historia de la química. Además se editaron siete volúmenes de la Colección Estudios de Historia Social de las Ciencias Químicas y Biológicas, dos volúmenes de los Anais da Sociedade Brasileira de História da Ciência y un volumen de la Collection de Travaux de l'Académie Internationale d'Histoire des Sciences.

Los logros que acabo de referir fueron posibles gracias a la gran capacidad, entusiasmo, interés, esfuerzo y compromiso sostenido por el núcleo de investigadores que conforman la directiva de la RIHECQB, quienes supieron sumar a un número cada vez mayor de colegas y alumnos que nos han honrado con su participación en esta empresa intelectual colectiva tan exitosa.

Todo mi reconocimiento, admiración, respeto y amor a mis queridos compañeros de aventura. Gracias por brindarnos generosamente su inmensa sabiduría. Gracias por arroparnos con su gran fortaleza e integridad. Gracias por su amistad. Mi más profundo agradecimiento a las doctoras Ana María Alfonso Goldfarb, Celina Lértora, Ana Luisa Janeira, Luzia Aurelia Castañeda, Marcia Mendes Ferraz y Ana María Huerta, así como a los doctores Javier Puerto Sarmiento y Patrice Bret.

No puedo enumerar por falta de espacio a todas las personas e instituciones que nos han apoyado a lo largo de los años. Sin embargo debo de hacer una mención especial al trabajo realizado por los doctores José Luiz Goldfarb y Marcia Mendes Ferraz en la edición de los dos volúmenes de los Anais de la Sociedade Brasileira de História da Ciência; a la maestra Alba Morales y al Dr. Adolfo Olea por su valiosa colaboración en la edición de los últimos tres volúmenes de los Estudios de Historia Social de las Ciencias Químicas y Biológicas, así como al Dr. Gérard Empotz por la edición del libro Between the Natural and the Artificial: Dyestuffs and Medicines. A ellos, a los presentes y los ausentes les damos las gracias por tantos años de enseñanzas, y por habernos colmado con su valioso apoyo y amistad. 
(Página deixada propositadamente em branco) 


\section{Colecçãa \\ 2 Ciências e Culturas Caimbra 2006}

